\title{
Control of topography, stress and diffusion at
} molecule-metal interface.

Nikolai B. Zhitenev ${ }^{1,5}$, Weirong Jiang ${ }^{1,2}$, Artur Erbe ${ }^{3}$, Zhenan Bao $^{4}$, Eric Garfunkel $^{2}$, Donald M. Tennant ${ }^{1}$ and Raymond A. Cirelli ${ }^{1}$

${ }^{1}$ Bell Labs., Lucent Technologies, Murray Hill, New Jersey 07974

${ }^{2}$ Department of Chemistry, Rutgers University, Piscataway, New Jersey 08854

${ }^{3}$ Fachbereich Physik, Universität Konstanz, 78464 Konstanz, Germany

${ }^{4}$ Department of Chemical Engineering, Stanford University, Stanford, California 94305

email: zhiten@lucent.com

\begin{abstract}
.
Transport properties of metal-molecule-metal junctions containing monolayer of conjugated and saturated molecules with characteristic dimensions in the range of 30-300 $\mathrm{nm}$ are correlated with microscopic topography, stress and chemical bonding at metalmolecule interfaces. Our statistically significant dataset allows us to conclude that the conductivity of organic molecules $\sim 1.5 \mathrm{~nm}$ long is at least 4 orders of magnitude lower than is commonly believed.
\end{abstract}

${ }^{5}$ Corresponding author 
PACS: 73.20.-r, 73.22.-f, 73.40.Gk, 73.40.Rw, 73.63.Rt

\section{Introduction}

Reliable and scalable integration of organic molecules within nanoscale electronic devices has the potential to dramatically expand available device functionality. Similar to other device platforms such as Si-based technology, the electronic properties of devices that are just a few atomic layers thick are determined not solely by the properties of the host material but are equally dependent on dopants, defects and electronic states at interfaces. Incorporation of molecules in small devices calls for the simultaneous solutions of many interrelated material, electronic and chemical issues. In this paper, we focus on devices built of self-assembled molecular layers (SAM) enclosed between two metal electrodes[1-3]. Typically, organic molecules do not have electronic states at the energy close to the Fermi energy of common metals. Electrons travel between metal electrodes by tunneling through the molecules in the gap between highest occupied (HOMO) and lowest unoccupied (LUMO) molecular orbitals. Tunneling transmission is governed by the energy difference between the Fermi level of the contacts and the closest

molecular orbital and by the spatial extent of the molecular orbital. Because of the exponential sensitivity of tunneling transmission to energy and distance, the conductance of real devices can be much larger or much smaller than that from molecular tunneling.

A single state in a molecular layer with defect energy close to the Fermi energy can dramatically increase the transmission. The most common origin of defects in molecular devices is penetration of metal contacts into the molecular layer. During and after fabrication, metal filaments can form, either completely shorting the source and the drain 
electrodes and limiting the yield of useful devices[4, 5], or strongly increasing device conductance[6]. Metal particles can penetrate into the molecular layers[7]. In the process of the metal contact film growth and crystallization the electrode can protrude into the layer, thus deforming molecules and affecting conductance[8]. On the other hand, distorted chemical bonds at the interface between metal and molecule can decrease the tunneling transmission. Metal films commonly used for electrodes, e.g., Au, Ag, Ti, Pt grow and interact very differently on top of molecular layers[9-12]. Currently, every experiment is unique in that the atomic placement of the relevant device constituents cannot be determined accurately.

The main goal of the paper is to study the phenomena affecting the conductance of molecular devices by systematically varying the growth conditions at the metal-molecule interface. Specifically, first we optimize the surface topography of the metal electrode used for the assembly of molecular layer reducing the density of structural defects in the SAM. This dramatically reduces the diffusion of the top metal contact through the molecular layer, increasing the device yield to $>90 \%$. Then, we experiment with chemical bonding and surface topography at the top metal-molecule interface.

\section{Experimental details}

The general approach for molecular device fabrication is to perform the most critical patterning of nanometer features without molecules, assemble the molecules, and to complete the structure with a relatively non-invasive processing step. We use small shadow masks defined within a stack of $\mathrm{SiO}_{2} / \mathrm{SiN}_{\mathrm{x}} / \mathrm{SiO}_{2}$ layers grown on degenerately doped $\mathrm{Si}$ substrates to obtain features below the lithographic limit[5, 13-16]. The 
fabrication of metal-SAM-metal junctions using the masks is illustrated in Figure 1. First, bottom electrodes are defined by evaporation. In the current study, the bottom electrode is $\mathrm{Ti} / \mathrm{Au}(5 \mathrm{~A} / 300 \mathrm{~A})$. The electrodes are separated by a bridge with a width in the range of 100-300 nm. Next, a SAM is deposited from solution in the usual manner. Two types of molecules, representing opposite ends of expected electronic functionality, are used in this study. Terthiophenedithiol (T3), synthesized using previously described methods[17], is a conjugated molecule with thiol groups responsible for chemical attachment to metal electrodes. Decanedithiol (C10) is a fully saturated molecule with a length of $1.5 \mathrm{~nm}$ similar to T3 length. The substrates with bottom $\mathrm{Au}$ electrodes were soaked in a tetrahydrofuran (THF) solution of the thiols (about $0.01 \mathrm{mM}$ ) at room temperature for 24 hours, then rinsed with THF, toluene and isopropanol. Both molecules form SAMs with thiol terminations exposed at the top interfaces as proven by nanotransfer experiments[18]. Finally, a top electrode is evaporated through the same mask from a different angle. The size of the junction is controlled by the size of the bridge and the angle of the second evaporation. SEM images of representative junctions are shown in Figure 1c. A single chip contains 84 separate devices, allowing us to perform statistical analysis on nominally equivalent junctions and to vary the junction sizes. The electrical characterization does not require the removal of the mask stack or metal accumulated on top surface of the mask, thus minimizing this potential source of damage or contamination.

\section{Results.}


In the first experiments, we assembled both molecules on an as-deposited Au electrode. The top electrode was an $8 \mathrm{~nm}$ Au or Ag film. Resistance of all junctions appeared to be indistinguishable from the leads resistance which is in the range of $300 \Omega-1 \mathrm{k} \Omega$ for devices with different lead geometries. We conclude that the evaporated metal penetrates through the SAMs shorting the devices. These results are consistent with our earlier observation of a low yield of non-shorted multi-grain junctions formed with different nano-templates[19]. The evaporated top metal easily diffuses near defects in molecular packing induced by multiple grain boundaries of the bottom electrode.

In the next set, we anneal ( $250 \mathrm{C}, 5 \mathrm{~min})$ the chips after deposition of the bottom $\mathrm{Au}$ contact. The annealing modifies the grain structure of polycrystalline Au film, making the grains smother and the grain size larger. The distribution of device resistances measured at room temperature is shown in Fig. 2a. Although the variation is very broad, the yield of non-shorted devices is above $\sim 96 \%$ for T3 and $\sim 92 \%$ for C10 SAMs; this is much higher than in previous experiments by others $(0.5 \%-5 \%)$ with similar electrode arrangements $[4,5,15]$. Surprisingly, the apparent median resistance for T3 devices is higher than for $\mathrm{C} 10$ devices, contrary to all expectations. This clearly shows that the conductance of real junctions is not directly determined by the electronic structures of the molecules. No scaling of the resistance with the junction area is observed.

To study bond formation at the top interface, we used overlayers of metals with different chemical reactivities. Au, Ag or Ti (7nm) were studied (Figure 2). Silver is more reactive than $\mathrm{Au}$, and titanium is known to strongly react not only with thiol groups at the top interface but also with carbon atoms[12]. The electrical properties of T3-Ag junctions are generally similar to those of T3-Au junctions. A significant percentage of the $\mathrm{C} 10-\mathrm{Ag}$ 
junctions were shorted. The conductance of the junctions with $\mathrm{Ti}$ overlayers is consistently higher. The overall histograms are very similar for both T3 and C10 SAMs.

The microscopic topography at the top SAM-metal interface is generally unknown. In an attempt to control the topography, we intentionally create clusters at the top interface. First, $0.3-0.5 \mathrm{~nm}$ of $\mathrm{Au}$ is evaporated on top of the SAM, simultaneously on the chips and on reference SAMs assembled on atomically flat Au substrate. The evaporation chamber is vented, and the reference sample is used to examine the topography of the overlayer by STM. Clusters with average diameter $\sim 6 \mathrm{~nm}$ are clearly seen (Figire $3 \mathrm{f}$ ). The junction fabrication is completed by evaporating an $8 \mathrm{~nm}$ thick film of Au. The electrical properties of the junctions changes dramatically in comparison with the uninterrupted $\mathrm{Au}$ evaporation as shown in Figure 3a and 3b. All T3 junctions are shorted while all C10 junctions are highly resistive (for convenience, we lump all $\mathrm{R}>10^{12} \Omega$ in a single bin).

In the next experiment, after deposition of $0.5 \mathrm{~nm}$ of $\mathrm{Au}$, the evaporation is interrupted for $3 \mathrm{~min}$ followed by continuous evaporation of $8 \mathrm{~nm}$ of Au (Figure $3 \mathrm{c}$ and $3 \mathrm{~d}$ ). The results clearly fall between the continuous Au evaporation shown in Figure 2 and the previous experiment. Half of the $\mathrm{T} 3$ junctions are not shorted, and the $\mathrm{C} 10$ junctions are less resistive on average. The shorted T3 junctions can be electrically driven into a more resistive state. Voltage pulse with $\sim 30-50 \mathrm{mV}$ amplitude and rise time below $1 \mu$ s usually triggers modification of the shorted junctions. We note that applying de voltage up to $1 \mathrm{~V}$ does not change the junction conductance. The distribution of resistances of junctions after the breakdown is shown in Figure 3e.

Junction resistance measured at room temperature only partly characterizes the transport properties. We studied conductance of the junctions as a function of source- 
drain voltage and temperature. The specific details of current-voltage (I-V) curves vary significantly as can be expected from the broad distribution of conductance values. Some representative results are illustrated in Figure 4.

Transport characteristics of all $\mathrm{T} 3-\mathrm{Ti}$ and $\mathrm{C} 10-\mathrm{Ti}$ junctions are quite similar (Figure 4a). Conductance falls only by $5-15 \%$ as temperature changes from $300 \mathrm{~K}$ to $4.2 \mathrm{~K}$. Lowtemperature $\mathrm{dI} / \mathrm{dV}$ curves are rather smooth, with a small dip near zero. The lower conductance, the zero-bias dip and the smooth $\mathrm{dI} / \mathrm{dV}$ variation clearly differentiate these junctions from shorted ones. Weak temperature dependence and zero-bias anomaly are the usual signatures of tunneling conductance. Most of the non-shorted C10-Ag and all $\mathrm{C} 10-\mathrm{Au}_{\mathrm{clust}}$ junctions also display insignificant temperature dependence but the tunneling conductance in this case is lower by orders of magnitude in comparison with Ti overlayer junctions. A small percentage of other types of junctions also display weak temperature dependence of conductance.

The transport properties of majority of other junctions with $\mathrm{Au}$ and $\mathrm{Ag}$ overlayers are more complicated. I-V curves are usually linear at room temperature. Conductance measured at zero source-drain bias falls noticeably with decreasing temperature (Figure 4b). Low-temperature I-V curves display a non-linear region with characteristic voltage scale $\mathrm{V}_{\mathrm{sd}} \sim 25-200 \mathrm{mV}$. This general behavior is typical for T3-Au, C10-Au, T3-Ag, T3$\mathrm{Au}_{\text {clust }}$ junctions. Similar behavior has been observed in our previous study of multi-grain junctions fabricated on the tips[19]. Two separate conductance mechanisms contribute to the overall electrical transport. The temperature dependent part of the conductance can be identified as hopping transport, characterized by a small energy scale in the range $\sim 10$ $150 \mathrm{meV}$. The residual conductance observed at low temperature is a combination of 
direct tunneling between contacts, sequential tunneling through low-energy defect states and hopping. The relative contributions of hopping and tunneling to the overall conductance vary broadly from sample to sample.

Finer differences between transport behavior in T3 and C10 SAMs can be seen in the low-temperature dI/dV curves. Conductance peaks shown in Fig. $4 \mathrm{~b}$ are observed in most

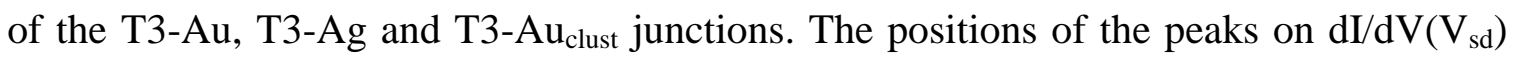
curves can be shifted by gate voltage in the case of T3-Au and T3-Ag junctions. Such behavior is reminiscent of single-electron charging of isolated islands. In $\mathrm{T} 3-\mathrm{Au}_{\mathrm{clust}}$ junctions, the peaks are stronger relative to the smoother background while the peak positions are usually insensitive to the gate voltage. No similar conductance peaks were observed in the majority of C10-Au samples.

\section{Discussion}

First, we comment on overall conductance values through the SAM. Commonly accepted[20] tunneling conductance per conjugated molecule of comparable length[21, 22] is $10^{-6}-10^{-8} \Omega^{-1}$ and conductance per alkane molecule[23] is $10^{-8}-10^{-9} \Omega^{-1}$. A median junction $\sim 100 \mathrm{~nm}$ by $100 \mathrm{~nm}$ contains $\sim 5^{*} 10^{4}$ molecules. If one assumes that every molecule is well-bonded on both sides, the median resistance of T3 junctions is $20 \Omega-2$ $\mathrm{k} \Omega$ and $2-20 \mathrm{k} \Omega$ for $\mathrm{C} 10$ junctions. If we conservatively assume having just a single bond per metal cluster of the top contact, a representative junction contains $\sim 300$ wellbonded molecules. The corresponding resistance estimates are $3 \mathrm{k} \Omega$ - $300 \mathrm{k} \Omega$ for $\mathrm{T} 3$ junctions, and $300 \mathrm{k} \Omega-3 \mathrm{M} \Omega$ for $\mathrm{C} 10$ junctions. Clearly, the results show that the tunneling conductance of molecules is lower by 4-6 orders of magnitude for both 
conjugated and saturated molecules, in strong contrast to the majority of previous calculations and experiments[20].

In fact, in the vast majority of devices we cannot single out the conductance associated with tunneling through the molecular orbitals. High values of tunneling conductance measured in the junctions with $\mathrm{Ti}$ overlayers are unlikely related to the electronic structures of the original molecules since no difference between conjugated and saturated molecules is seen in the experiment. Evaporated Ti reacts strongly with organics[12] attacking the SAM and forming Ti carbides and oxycarbides. Tunneling conductance measured in all other junctions is likely to be determined by the microscopic configuration of metal electrodes that have partly penetrated the SAM, and/or a small number of defect states rather than by molecular states.

The essential material transformations defining the electronic properties of molecular junctions are schematically illustrated in Figure 4c. Structural defects in SAMs assembled on as-deposited Au allow for easy diffusion of the top metal electrode through the layer. The SAM structure is significantly improved by annealing Au films before the deposition of molecules. The high yield of non-shorted devices achieved by evaporation of Au on top of SAMs suggests that practically all incident $\mathrm{Au}$ atoms are stopped at the top interface. The overall electrical properties of junctions formed with T3 and C10 SAMs are rather similar. We believe that during its growth and crystallization the Au overlayer penetrates deep into the SAM, exerting substantial pressure and deforming the molecules. The microscopic details of the electrode topography are almost independent of the molecule type in this particular case. Diffusion and growth of Ag overlayers differ depending on the SAM type. Ag penetrates alkane SAMs more easily. Finally, evaporated 
Ti can strongly react with SAMs, modifying electronic structure with little discrimination between conjugated and saturated molecules.

Relaxation of the thin Au overlayer accumulated at the top molecular interface and cluster formation results in two new phenomena. First, comparing properties of the junctions formed on C10 SAMs, we suggest that this relaxation significantly reduces stress at the interface and the penetration of the top metal contact into the SAM volume. The $\mathrm{C} 10$ junctions with a relaxed top interface are so resistive that their conductance cannot be reliably differentiated from a possible leakage through the substrate. Very different behavior is seen on conjugated SAMs. Effectively, the clusters diffuse through the SAM easier than separate Au atoms impinging the SAM during the evaporation. The cluster diffusion has to proceed along with a redistribution of molecules in the SAM. Apparently, the configuration with the clusters partly or fully submerged into conjugated SAM lowers the total energy of the system. The clusters that form at the edge of a continuous $\mathrm{Au}$ top electrode and partly penetrate into the SAM can account for the systematic observation of conductance resonances sensitive to the gate voltage in $\mathrm{T} 3$ junctions.

The results clearly expose a variety of material transformations and self-organization processes occurring during integration of organic and inorganic components in nanoscale devices. This is the first experimental research that systematically correlates the electrical properties of SAM-based molecular devices with the microscopic details of the metalmolecule interface. We have demonstrated that the generation of defects can be dramatically reduced for certain combinations of metals and molecules by changing surface topography and growth conditions at the interface. 


\section{Acknowledgements}

We would like to acknowledge useful discussions with E. Chandross and D. Hamann.

\section{References}

1. Collier C P, Mattersteig G, Wong E W, Luo Y, Beverly K, Sampaio J, Raymo F M, Stoddart J F and Heath J R A 2000 [2]Catenane-Based Solid State Electronically Reconfigurable Switch Science 2891172.

2. Chen J, Reed M A, Rawlett A M and Tour J M 1999 Large on-off ratios and negative differential resistance in a molecular electronic device Science 2861550.

3. Chen Y, Jung G Y, Ohlberg D A A, Li X M, Stewart D R, Jeppesen J O, Nielsen K A, Stoddart J F and Williams R S 2003 Nanoscale molecular-switch crossbar circuits Nanotechnology 14462.

4. Lee T, Wang W, Zhang J J, Su J, Klemic J F and Reed M A 2005 Cross-platform characterization of electron tunneling in molecular self-assembled monolayers Current Appl. Phys. 5213.

5. Austin M D and Chou S Y 2003 Fabrication of a molecular self-assembled monolayer diode using nanoimprint lithography Nano Lett. 31687.

6. Lau C N, Stewart D R, Williams R S and Bockrath M 2004 Direct observation of nanoscale switching centers in metal/molecule/metal structures Nano Lett. 4569.

7. Philipp G, Muller-Schwanneke C, Burghard M, Roth S and von Klitzing K 1999 Gold cluster formation at the interface of a gold/Langmuir-Blodgett film/gold microsandwich resulting in Coulomb charging phenomena J. Appl. Phys. 853374.

8. Son K-A, Kim H I and Houston J E 2001 Role of Stress on Charge Transfer through Self-Assembled Alkanethiol Monolayers on Au Phys. Rev. Lett. 835357.

9. Ohgi T, Sheng H-Y, Dong Z-C, Nejoh H and Fujita D 2001 Charging effects in gold nanoclusters grown on octanedithiol layers Appl. Phys. Lett. 792453.

10. Ohgi T, Fujita D, Deng W, Dong Z C and Nejoh H 2001 Scanning tunneling microscopy and X-ray photoelectron spectroscopy of silver deposited octanethiol selfassembled monolayers Surf. Sci. 493453.

11. Boer B d, Frank M M, Chabal Y J, Jiang W, Garfunkel E and Bao Z 2004 Metallic contact formation for molecular electronics: interactions between vapordeposited metals and self-assembled monolayers of conjugated mono- and dithiols Langmuir 201539. 
12. Walker A V, Tighe T B, Stapleton J, Haynie B C, Upilli S, Allara D L and Winograd N 2004 Interaction of vapor-deposited Ti and Au with molecular wires Appl. Phys. Lett. 844008.

13. Fulton T A and Dolan G J 1987 Observation of single-electron charging effects in small tunnel junctions Phys. Rev. Lett. 59109.

14. Kagan C R, Afzali A, Martel R, Gignac L M, Solomon P M, Schrott A G and Ek B 2003 Evaluations and Considerations for Self-Assembled Monolayer Field-Effect Transistors Nano Lett. 3119.

15. Lee J O, Lientschnig G, Wiertz F, Struijk M, Janssen R A J, Egberink R, Reinhoudt D N, Hadley P and Dekker C 2003 Absence of strong gate effects in electrical measurements on phenylene-based conjugated molecules Nano Lett. 3113.

16. Zhitenev N B, Meng H and Bao Z 2002 Conductance of small molecular junctions Phys. Rev. Lett. 88226801.

17. Boer B d, Meng H, Perepichka D F, Zheng J, Frank M M, Chabal Y J and Bao Z 2003 Synthesis and characterization of conjugated mono- and dithiol oligomers and characterization of their self-assembled monolayers Langmuir 194272.

18. Jiang W R, Zhitenev N B, Bao Z, Meng H, Abusch-Magder D, Tennant D and Garfunkel E 2005 Structure and bonding issues at the interface between gold and selfassembled conjugated dithiol monolayers Langmiur 218751.

19. Zhitenev N B, Erbe A and Bao Z 2004 Single- and Multigrain Nanojunctions with a Self-Assembled Monolayer of Conjugated Molecules Phys. Rev. Lett. 92186805.

20. Salomon A, Cahen D, Lindsay S, Tomfohr J, Engelkes V B and Frisbie C D 2003

Comparison of electronic transport measurements on organic molecules Adv. Mater. 15 1881.

21. Kergueris C, Bourgoin J P, Palacin S, Esteve D, Urbina C, Magoga M and Joachim C Electron transport through a metal-molecule-metal junction 1999 Phys. Rev. B 5912505.

22. Heurich J, Cuevas J C, Wenzel W and Schon G 2002 Electrical transport through single-molecule junctions: From molecular orbitals to conduction channels Phys. Rev. Lett. 88256803.

23. Tomfohr J K and Sankey O F 2002 Complex band structure, decay lengths, and Fermi level alignment in simple molecular electronic systems Phys. Rev. B 65245105. 

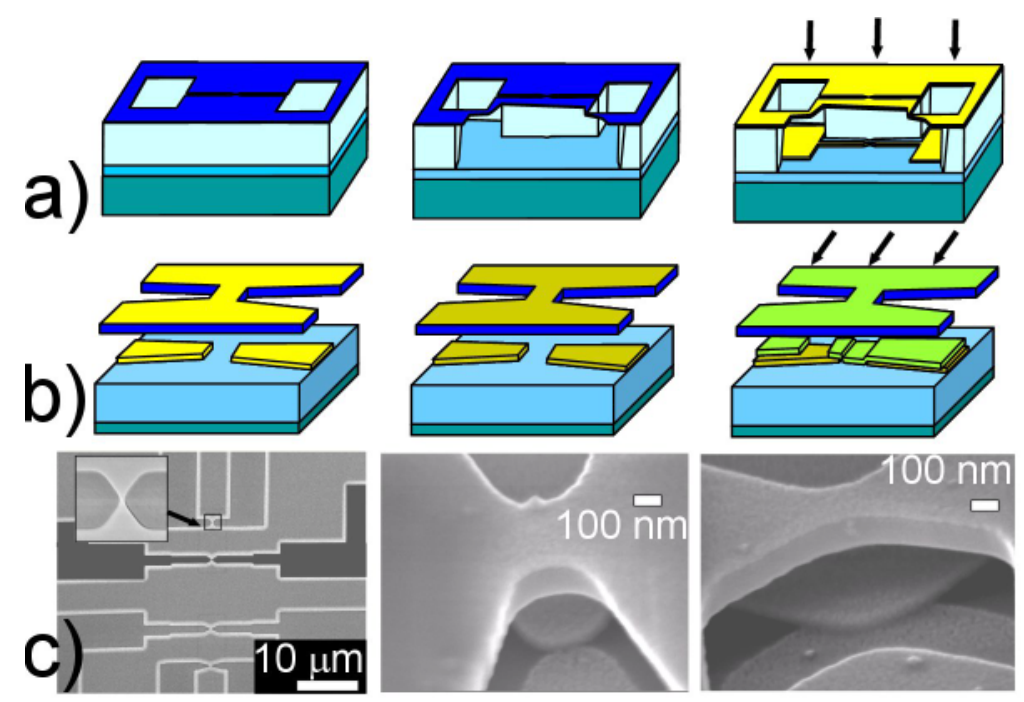

Figure 1. Fabrication of templates and molecular junctions. a). The stencil mask is defined within an insulating stack of $\mathrm{SiO}_{2} / \mathrm{Si}_{3} \mathrm{~N}_{4}$ layers grown on $\mathrm{Si}$ substrate. A layer of $\mathrm{SiO}_{2}$ (light blue, $10 \mathrm{~nm}$ in $5 \mu \mathrm{m}$ by $5 \mu \mathrm{m}$ central windows, $200 \mathrm{~nm}$ elsewhere on the wafer) is grown on $\mathrm{Si}$ (light purple) to isolate the substrate from the devices followed by $\mathrm{Si}_{3} \mathrm{~N}_{4}(\sim 400 \mathrm{~nm}$, cyan $)$ and $\mathrm{SiO}_{2}(\sim 150 \mathrm{~nm}$, blue) mask forming layers. The desired pattern is defined by photolithography and etching of the top $\mathrm{SiO}_{2}$ layer. $\mathrm{Si}_{3} \mathrm{~N}_{4}$ is selectively etched undercutting the top $\mathrm{SiO}_{2}$. The mask is used to define the pattern of evaporated metal electrodes (yellow). The metal accumulated on the top surface of the mask is electrically isolated from the evaporated electrodes. b) Fabrication of molecular junction using the template. Bottom electrodes are defined on the substrate by evaporation. Molecular layer is deposited from solution. Evaporation of second electrode from an angle completes the junction. c) SEM images of the template (one out of four shown samples is shaded) and examples of small and large junctions. 

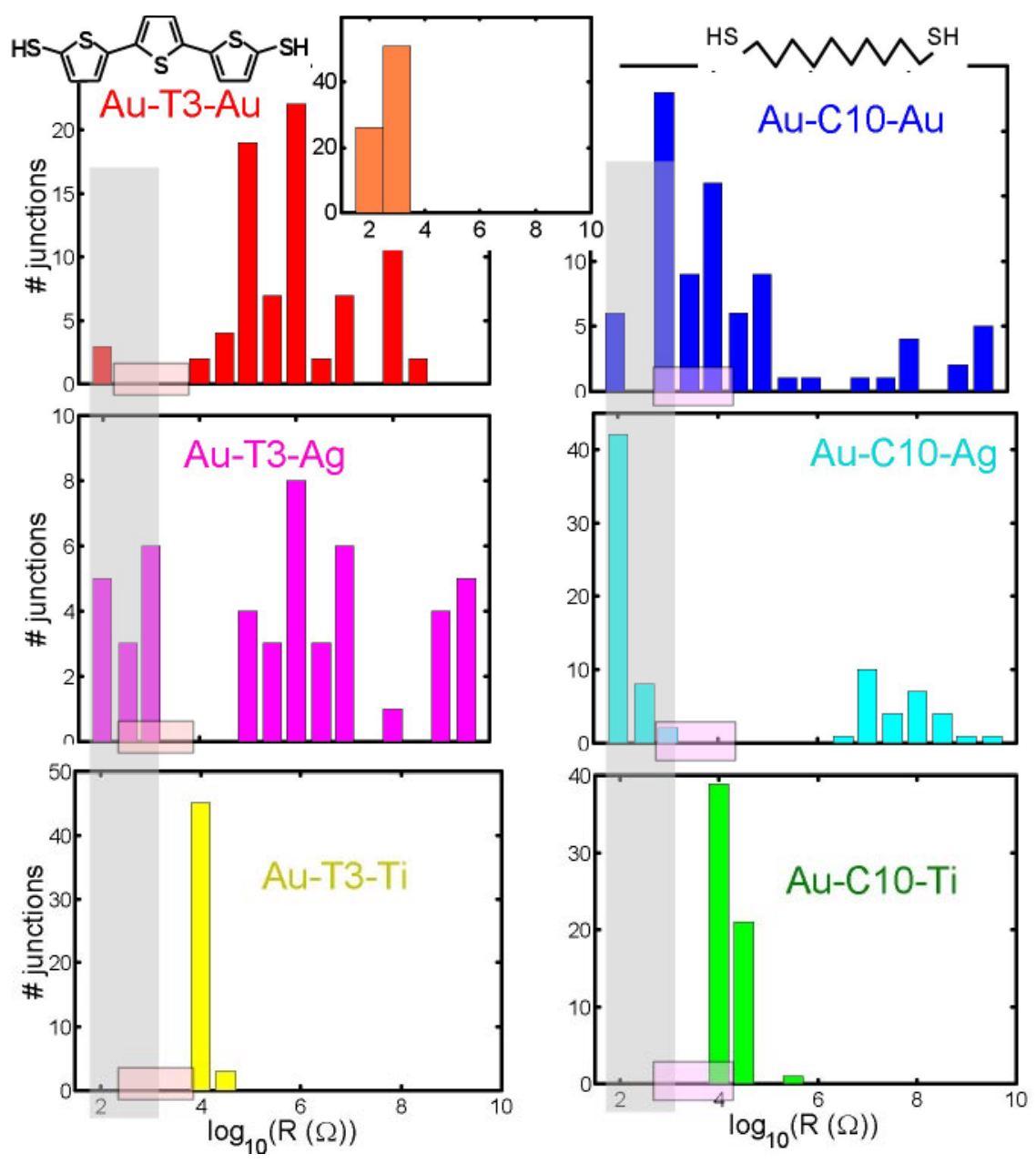

Figure 2. Distribution of junction resistances measured at room temperature. Resistance range corresponding to the leads resistance is gray colored (shorted junctions). Lightcolored bars show expected ranges of resistances assuming that (i) all molecules inside junctions are bonded to both electrodes and (ii) tunneling conductance of $10^{-6}-10^{-8} \Omega^{-1}$ / T3 and $10^{-8}-10^{-9} \Omega^{-1} / \mathrm{C} 10$. Inset in the top row shows results for junctions fabricated on as-deposited Au electrode. 

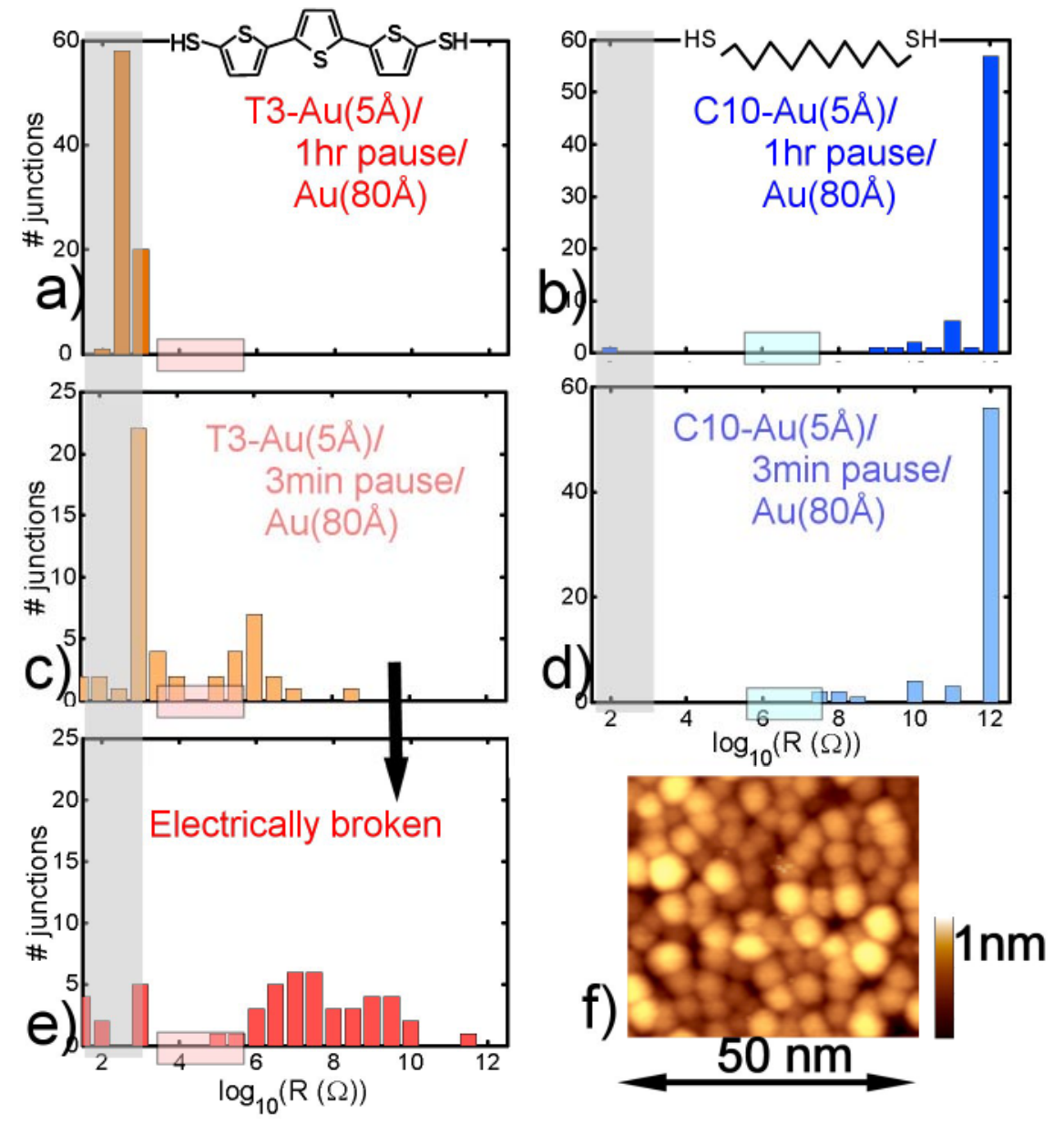

Figure 3. Distribution of junction resistances with the clusters created at the top metalmolecule interface. Top metal contact is formed by depositing $0.5 \mathrm{~nm}$ of Au film first, letting the film relax for time $\tau_{\text {rel }}$, and depositing another $8 \mathrm{~nm}$ of Au: a) $\tau_{\text {rel }}=1 \mathrm{hr}$, T3 $\mathrm{SAM} \mathrm{b)} \tau_{\text {rel }}=1 \mathrm{hr}, \mathrm{C} 10 \mathrm{SAM}$ c) $\left.\tau_{\text {rel }}=3 \mathrm{~min}, \mathrm{~T} 3 \mathrm{~d}\right) \tau_{\text {rel }}=3 \mathrm{~min}, \mathrm{C} 10$. e) distribution of junction resistance from (c) after the soft electrical breakdown. Colored bars show expected range of resistances assuming that (i) only one molecule per cluster forms good bonds at both ends and (ii) tunneling conductance of $10^{-6}-10^{-8} \Omega^{-1} / \mathrm{T} 3$ and $10^{-8}-10^{-9} \Omega^{-}$ 1/C10. f) STM image of clusters formed on top of T3 SAM. 


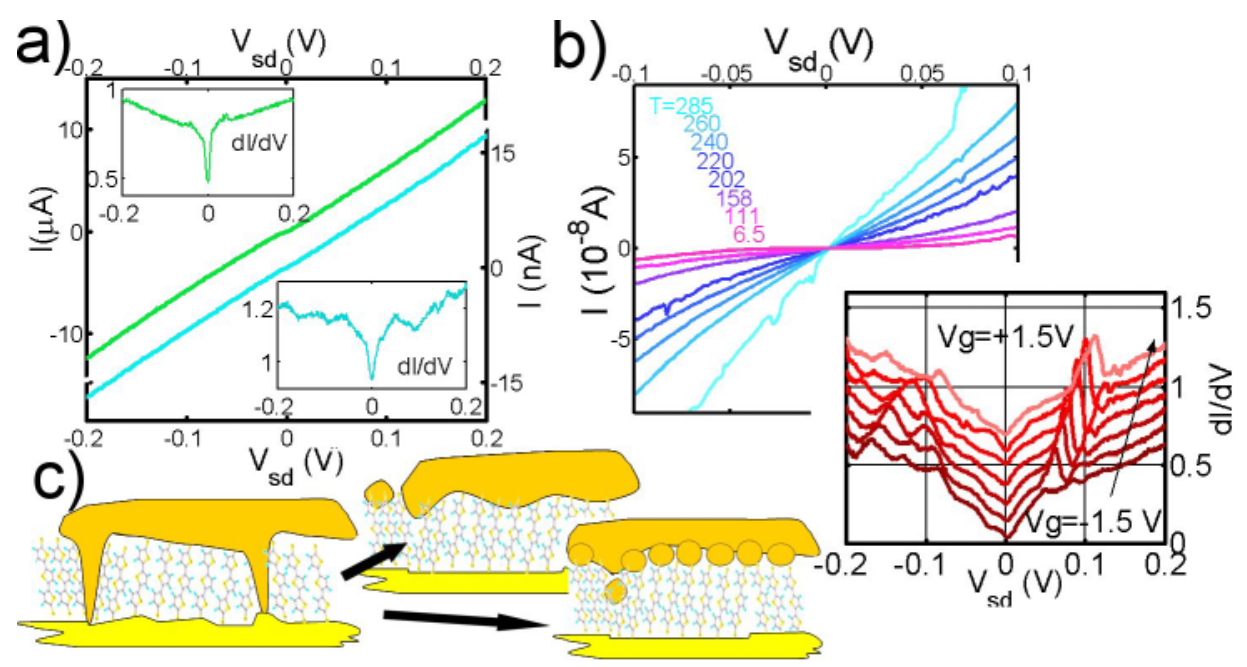

Figure 4. Examples of characteristic transport behavior. a) IV curves measured on C10Ti (top) and C10-Ag (bottom) junctions. The current scales are different. T= $8 \mathrm{~K}$. Insets: Corresponding differential conductance $\mathrm{dI} / \mathrm{dV}$ as a function of $\mathrm{V}_{\mathrm{sd}} \mathrm{b}$ ) IV curves measured at different temperatures on $\mathrm{C} 10-\mathrm{Au}$ junction. Inset: Set of dI/dV curves measured on T3$\mathrm{Au}$ junction at different gate voltages. $\mathrm{T}=8 \mathrm{~K}$. c) Schemes of metal assembly and penetration into molecular layer illustrating material modifications and interface topography studied in this paper. Junctions assembled on rough Au surface are usually shorted because of easy metal diffusion along structural defects of the SAM. Yield of non-shorted junctions is high if bottom $\mathrm{Au}$ electrode is annealed before the SAM deposition. Metal electrodes can protrude into the SAM volume and/or deform molecules. Relaxation of thin metal underlayer reduces stress at the interface. 